\section{Color name as a function of surround luminance and stimulus duration*}

\author{
MICHAEL H. SIEGEL and ANNE B. SIEGEL \\ Albion College, Albion, Michigan 49224
}

A color-naming experiment was performed in which both surround luminance and exposure duration were varied. The data showed substantial effects from these changes; however, none could be interpreted to indicate the presence of a Bezold-Brucke shift or tritanopia.

In the past few years there has been a great deal of attention directed to studies of color naming (Bouman \& Walraven, 1957; Marriott, 1959; Beare, 1963; Boynton, Schaefer, \& Neun, 1964). Few have dealt with how stimulus duration influences color appearance (Weitzman \& Kinney, 1967; Kaiser, 1968). Only one has been concerned directly with investigating the relationship of varying stimulus intensity and duration with color naming (Luria, 1967). In his careful study, Luria employed the method suggested by Boynton et al (1964) to study color naming at 11 selected wavelengths whose intensities were varied from .15 to $15 \mathrm{fL}$ and whose exposure duration was varied from 2 to $300 \mathrm{msec}$. Luria discovered both the presence of a Bezold-Brucke shift with changes in intensity and duration and a "low-energy tritanopia." Both Weitzman and Kinney (1967) and Kaiser (1968) have also reported a tritanopia-like effect with small, briefly presented stimuli.

The object of the present study was to attempt a systematic investigation of the effects of both exposure duration and surround luminance upon color naming. The questions of whether or not a Bezold-Brucke effect and tritanopia could be demonstrated when brightness, and not stimulus intensity, varied were of major interest.

\section{Apparatus}

\section{METHOD}

$A$ Bausch and Lomb grating monochromator provided the stimulus, which was maintained at $.5 \mathrm{fL}$ throughout the study. The duration of the stimulus flash was controlled by a sector shutter that permitted various exposure times to be used. Calibrations of wavelengths and exposure duration were made both before and after experimentation. No change in experimental values was detected.

The $O$ sat in a separate booth and viewed the stimulus in Maxwellian view through a $3 \cdot \mathrm{mm}$ artificial pupil. The

*This research was supported in part by Public Health Service Grant MH-14281. stimulus, which subtended $3 \mathrm{deg}$ at the O's eye, appeared through a circular hole in white surround screen. This screen was by a light whose color temperature matched Illuminant $\mathrm{C}$ but whose luminance could be varied. All observations were made foveally with the right eye; the left eye was occluded. A head and chin rest was used to minimize eye movements.

\section{Observers}

There were three Os. All were color normal as defined by the Farnsworth-Munsell 100 hue test and $\mathrm{AO}$ pseudoisochromatic plates. All Os were emmotropic and were experienced in the kind of judgment required.

\section{Procedure}

The spectrum was sampled at $10-\mathrm{nm}$ intervals from 400 through $640 \mathrm{~nm}$, except that 5-nm intervals were used in the range $560-590 \mathrm{~nm}$ to ensure a more nearly complete sampling of the yellows. In all, a total of 28 wavelengths were tested. Three stimulus exposure times, $.02, .06$, and $.10 \mathrm{sec}$, were combined with three surround luminances, $.1, .5$, and $1.0 \mathrm{fL}$, for a total of nine conditions. All 28 wavelengths were tested in each condition. illuminated from above and behind the 0 surround luminances.
Os were instructed to respond verbally to the stimulus with one of 10 color categories; red, yellow-red, yellow, green-yellow, green, blue-green, blue, blue-red, white or gray, and "not sure." A session consisted of four mixed-order presentations of the 28 wavelengths at one time luminance condition. Each $O$ had three sessions at each of the nine conditions. The conditions were presented in a different random order to each 0 . Each session lasted about $50 \mathrm{~min}$.

\section{RESULTS}

Since there were no significant differences among $\mathrm{Os}$, data from all three have been combined. For each condition, responses at any wavelength were recorded according to color category, the number of responses in each category was counted, and this number was converted to a percentage of the total responses (36) at that wavelength. Data for each of the nine conditions are presented in Figs. 1-9. The white-gray and "not sure" categories are not plotted but were counted in obtaining the percent color name. In each figure, percent color name is plotted on the ordinate and wavelength on the abscissa. Each color name occupies a well-defined portion of the spectrum, but the amount that one color overlaps another and the height of the curves varies widely from one condition to another.

The first three figures show the effects of increments in surround luminance at an exposure duration of $.02 \mathrm{sec}$. As surround luminance increased and the stimulus became effectively dimmer, stimuli elicited responses in more than one category, and the range of wavelengths producing at least some responses in a particular category was correspondingly increased. These two tendencies typically produced a function that retained its characteristic shape, but

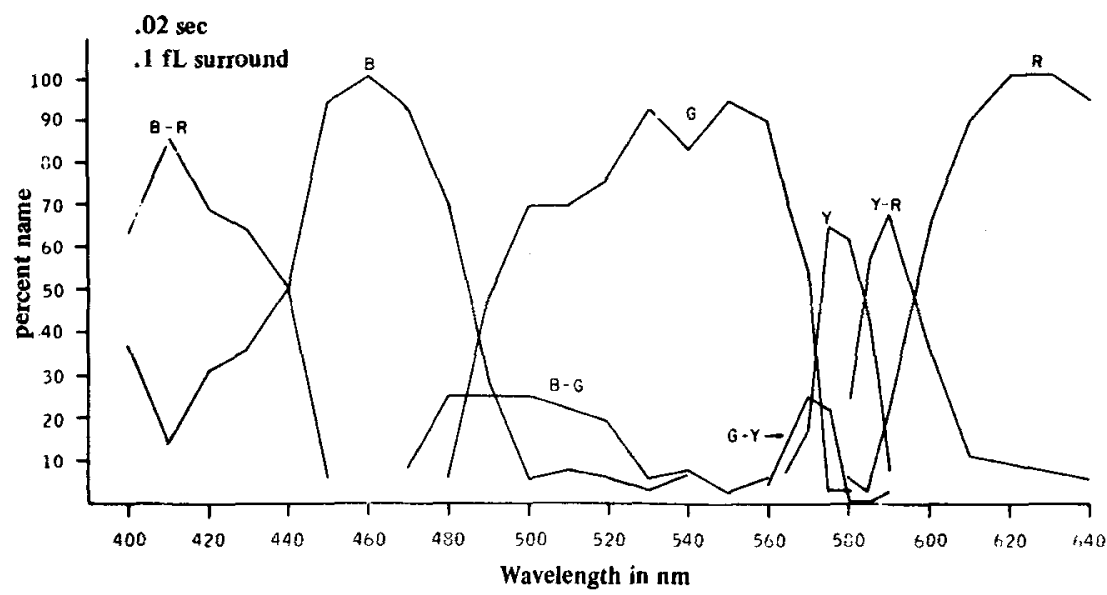

Fig. 1. Color name as a function of wavelength, .02-sec exposure duration, .1-fL 


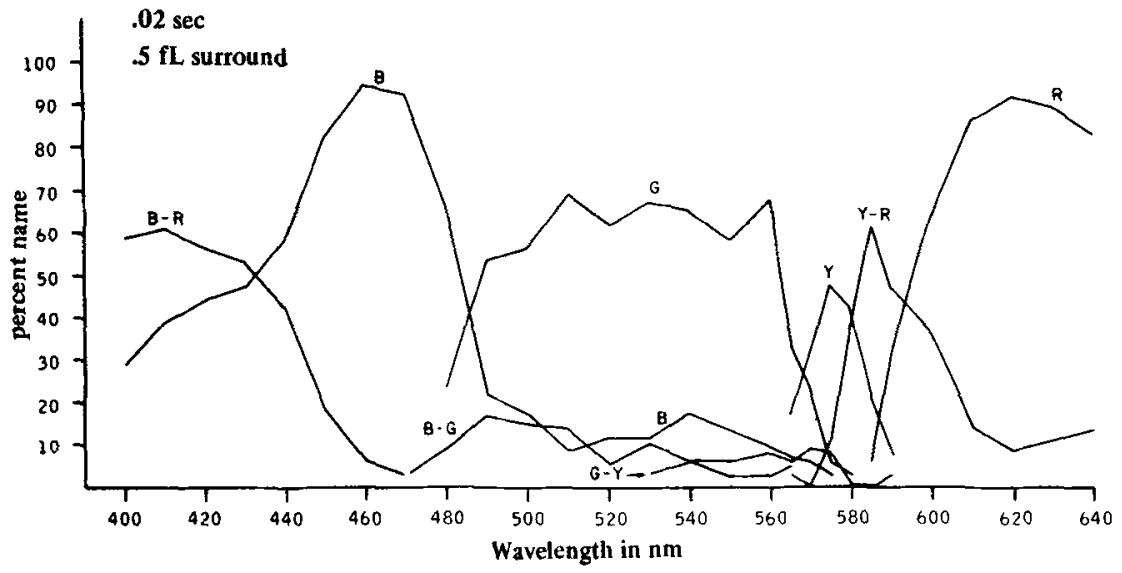

Fig. 2. Color name as a function of wavelength, .02-sec exposure duration, .5-fL surround luminances.

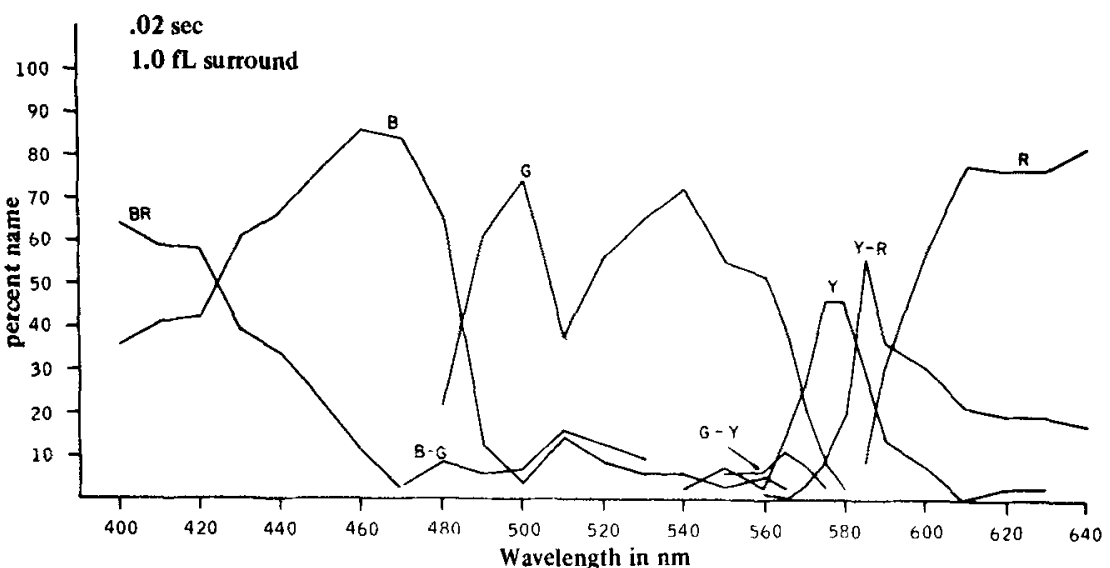

Fig. 3. Color name as a function of wavelength, .02-sec exposure duration, 1.0-fL surround luminances.

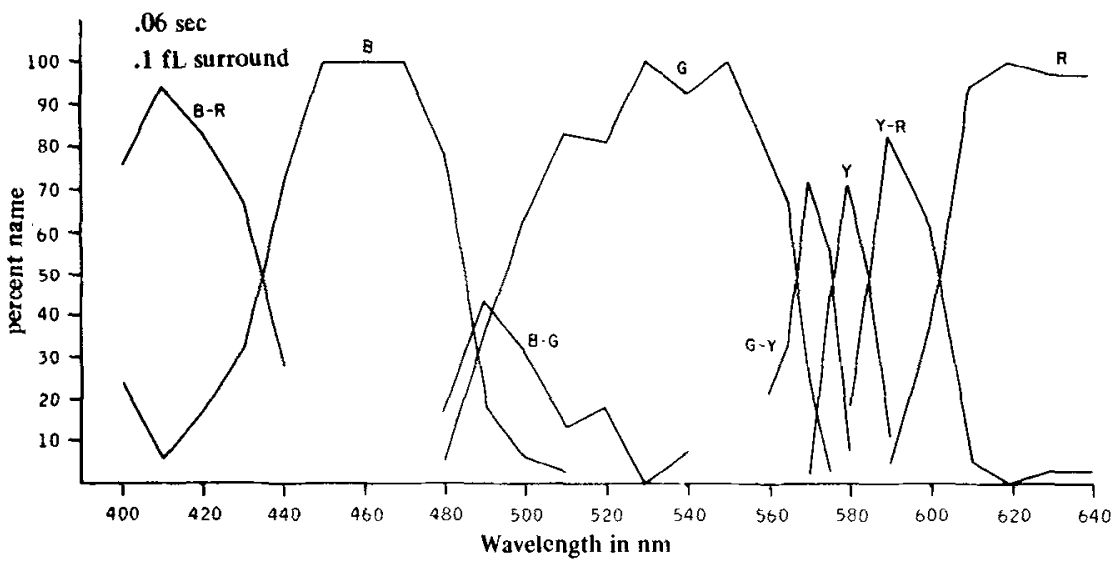

Fig. 4. Color name as a function of wavelength, .06-sec exposure duration, .1-fL surround luminances.

that decreased in height, increased in range, and often acquired a "tail" in one or both directions from the main portion of the curve. The first increase in surround luminance, from .] to $.5 \mathrm{fL}$ caused a substantial decrease in the height of the a tendency to become bimodal. In addition to changes in height, the wavelength location of the modal value of the green-yellow, yellow, and yellow-red categories was shifted toward shorter wavelengths, indicating that this portion of the spectrum appeared somewhat greener as surround luminance was increased. The second increase in surround luminance, from .5 to $1.0 \mathrm{fL}$ (Figs. $2 \& 3$ ) produced a tail on the yellow function in both directions and increased the height of the tail on the yellow-red function. The blue-green and green-yellow function decreased in range, indicating a decrease in the Os' use of these categories. Blue, yellow, yellow-red, and red continued to decrease in height and increase in range, and green became distinctly bimodal, with one peak at about $500 \mathrm{~nm}$ and one at $540 \mathrm{~nm}$, but there was no shift in the modal position of any category.

Figures 4, 5, and 6 show data for $.06 \mathrm{sec}$. As surround luminance was increased from .1 to $.5 \mathrm{fL}$, blue-red, blue, and green remained almost invariant. Green-yellow decreased in height but not in range, and yellow increased in height but not in range. This indicates a considerable decrease in the use of the green-yellow category and some increase in the use of the yellow. Two categories showed a change in wavelength position: blue-green peaked at a longer wavelength and yellow-red at a shorter one. When surround luminance was further increased to $1.0 \mathrm{fL}$, the height of the yellow and green-yellow functions decreased. Green increased in range but maintained the same height, indicating increased use of this category. The blue-green function returned to its original (Fig. 4) position of $490 \mathrm{~nm}$, and yellow shifted about $5 \mathrm{~nm}$ toward green.

At the longest exposure duration (Figs. 7,8 , and 9), response changes due to increased surround luminance were small. Blue-red, blue, and green were virtually unchanged at all surround luminance levels. At the lowest surround luminance level, yellow-red and red produced an atypical bimodal function, which disappeared at the other surround conditions. Green-yellow shifted about $5 \mathrm{~nm}$ toward longer wavelengths with the first increment in surround luminance, and blue-green decreased in height. The second increment decreased the height of green-yellow, yellow, and yellow-red, added "tails" to the blue-green and blue functions, but produced no shifts in wavelength postion.

In general, as the stimulus was dimmed by increasing surround luminance, the largest changes in response occurred with the first increase, from .1 to $.5 \mathrm{fL}$. These changes were primarily alterations in the height and range of the color function, 
which were especially pronounced in the blue-green, green-yellow, yellow, and yellow-red categories. There was also some tendency for the modal position of the yellow functions to be shifted slightly toward green. At $.02 \mathrm{sec}$, the first luminance increment shifted all three yellow categories slightly; at $.06 \mathrm{sec}$, yellow and yellow-red were shifted, but only after both increments; and, at .1 sec, only green-yellow was affected, and in this case it was moved toward yellow rather than green. Blue-red, blue, green, and red were very stable at all surround-luminance levels, although blue often acquired a "tail" and green showed some tendency to become bimodal at the higher luminance levels.

The data can also be compared for the effects of variation in exposure duration. When surround luminance is held constant at .1 fL (Figs. 1, 4, and 7), increasing exposure duration from .02 to $.06 \mathrm{sec}$ produced some increase in height and decrease in range for most functions, especially blue-green and the yellow group. Blue-green showed no distinct peak at $.02 \mathrm{sec}$, but a well-defined one at $.06 \mathrm{sec}$. Green-yellow, yellow, and yellow-red all increased in height at $.06 \mathrm{sec}$ and shifted toward longer wavelengths. Red peaked at about the same wavelengths $(630 \mathrm{~nm})$, but showed a wider range and considerably more area under the curve, indicating increased use of this category at shorter wavelengths. When exposure duration was increased again from .06 to $.1 \mathrm{sec}$, yellow-red and red became bimodal, and green-yellow and yellow-red ended abruptly. All functions tended to become smoother and more symmetrical with each increase in exposure duration.

Comparisons of exposure duration at $.5 \mathrm{fL}$ surround luminance (Figs. 2, 5, and 8) show large changes. Every category except red showed an increase in height and decrease in range as duration was increased from .02 to $.06 \mathrm{sec}$. The area under the blue-red curve increased greatly, the "tails" of the blue and yellow-red functions disappeared, the maximum green value increased from $70 \%$ to $95 \%$, and blue-green and green-yellow moved from long flat functions to much more narrow and sharp ones. Despite these substantial changes in height and range, only yellow shifted wavelength position, moving from 570 to $580 \mathrm{~nm}$. A second increase in exposure duration, however, caused only slight changes in height for any category, but altered the modal location of blue-green toward shorter wavelengths and that of green-yellow and yellow-red toward longer ones.

Increments in exposure duration at a surround luminance of $1.0 \mathrm{fL}$ (Figs. 3, 6,

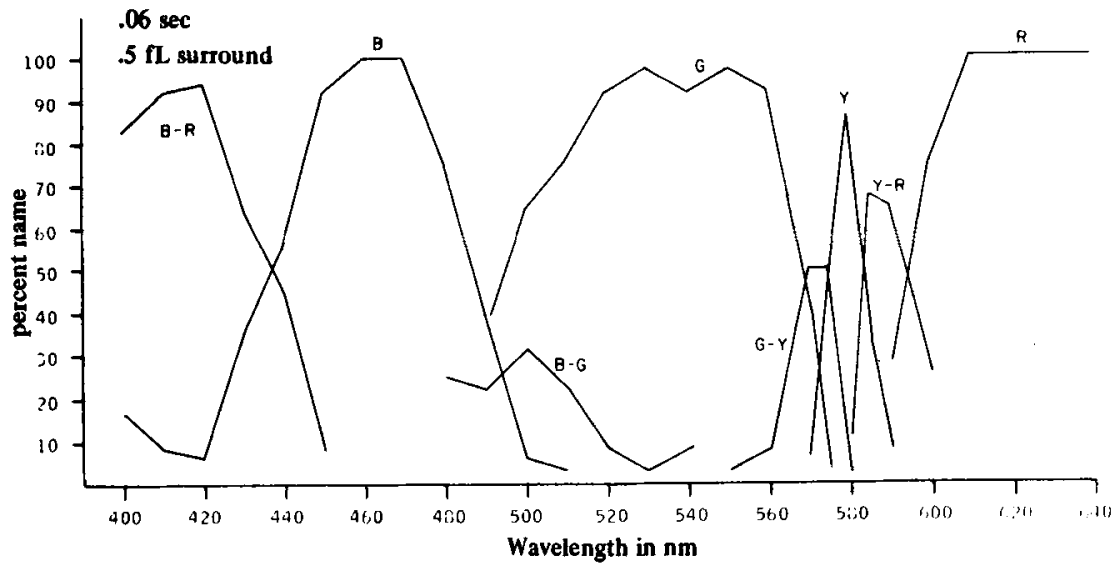

Fig. 5. Color name as a function of wavelength, .06-sec exposure duration, .5-fL surround luminances.

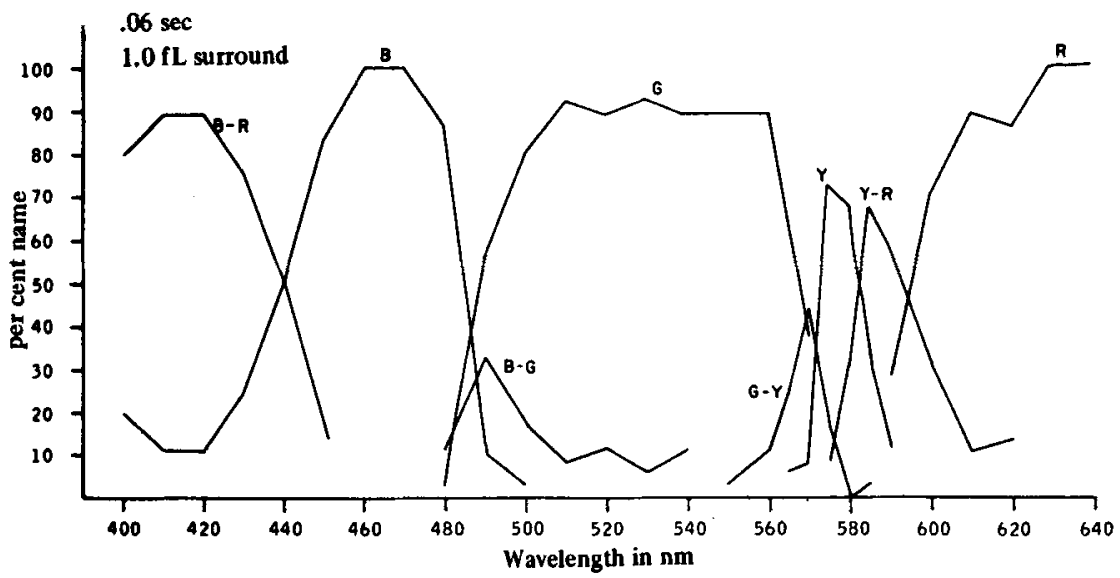

Fig. 6. Color name as a function of wavelength, .06-sec exposure duration, 1.0-fL surround luminances.

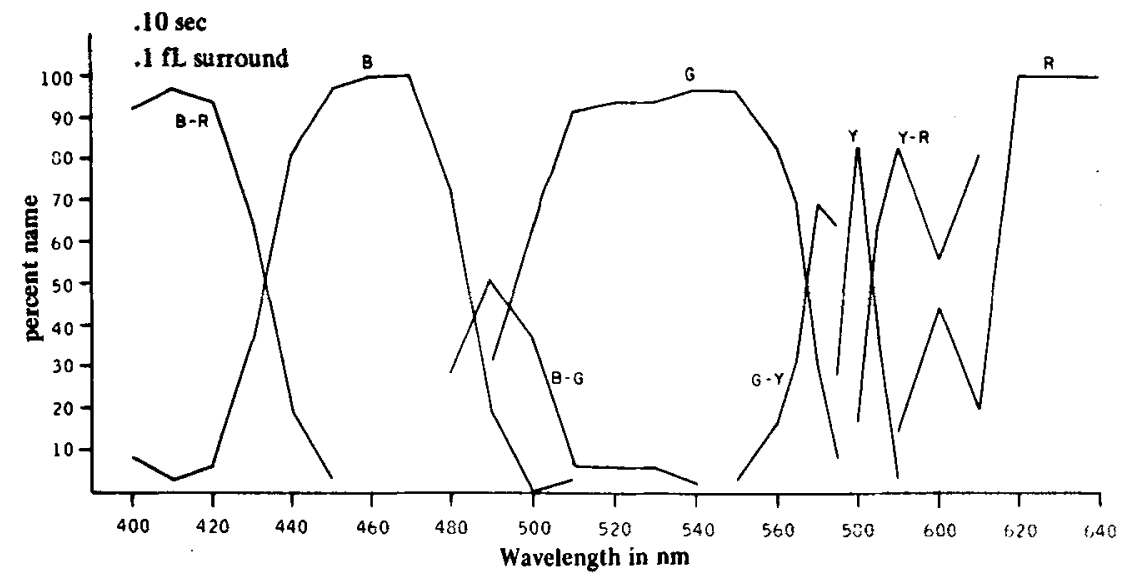

Fig. 7. Color name as a function of wavelength, .1-sec exposure duration, .1-fL surround luminances.

and 9) again produced radicai changes in shape for all color functions. Blue-red, blue, blue-green, green-yellow, yellow, and yellow-red all increased in height and decreased in range as duration was increased from .02 to $.06 \mathrm{sec}$. Green and red also increased in height, but main tained the same range, and green, which was bimodal at $.02 \mathrm{sec}$, assumed a more typical "plateau" shape. Blue-green and green-yellow altered the wavelength position of their modes, moving toward 


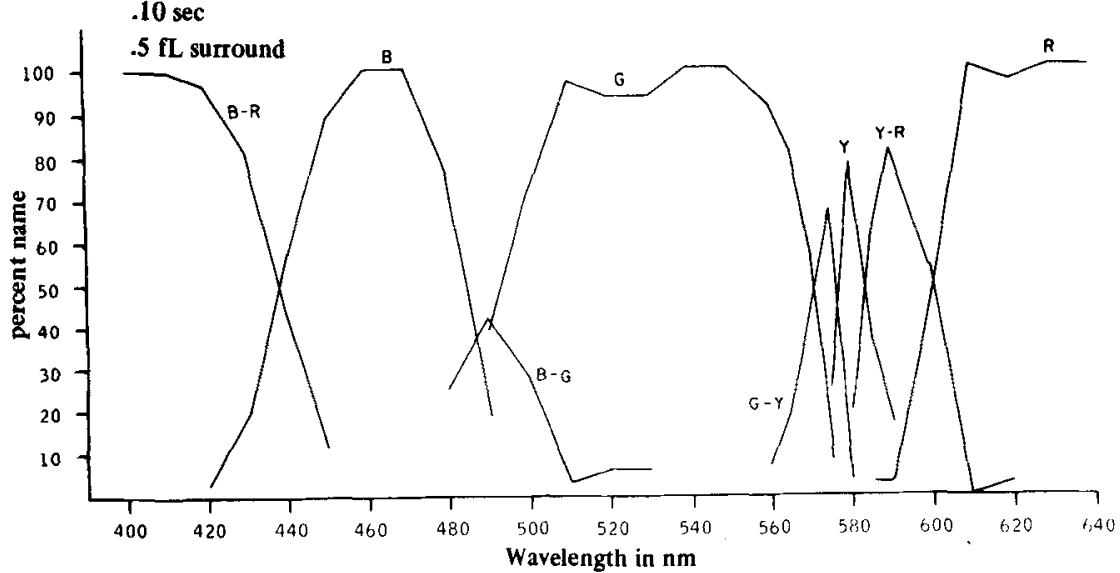

Fig. 8. Color name as a function of wavelength, .1-sec exposure duration, .5-fL surround luminances.

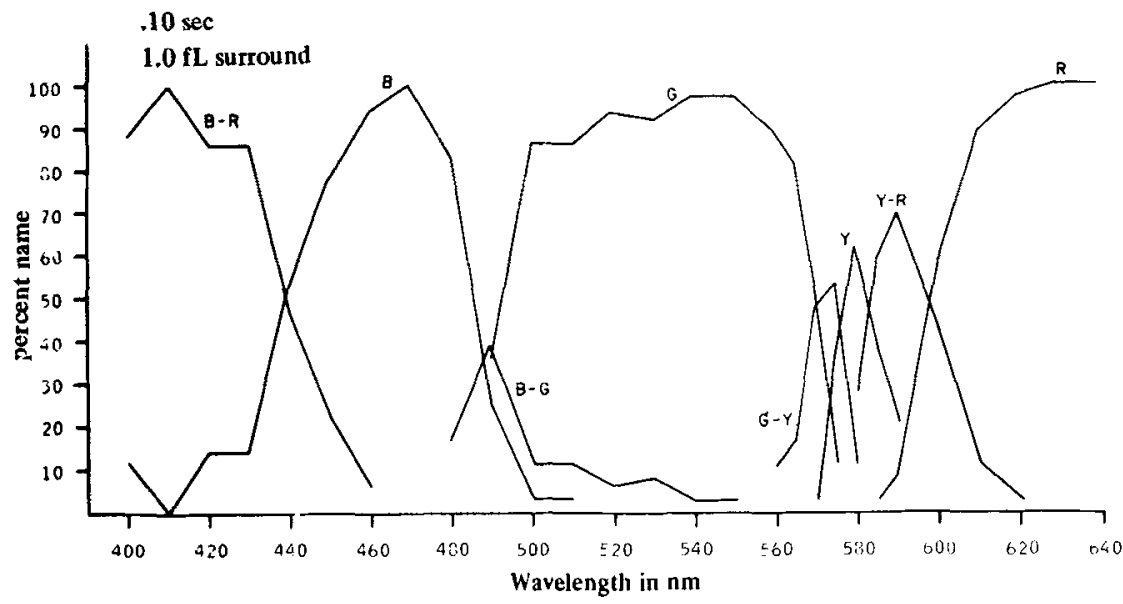

Fig. 9. Color name as a function of wavelength, .1-sec exposure duration, 1.0-fL surround luminances.

blue and yellow respectively, but this appears to be largely a result of their change from flat functions without any pronounced central tendency to sharp inverted "Vs" with clear modal locations. As exposure duration was increased again from .06 to $.10 \mathrm{sec}$, only blue-red and yellow showed much change in $1+\mathrm{ight}$, with blue-red increasing slightly and yellow decreasing somewhat; however, blue-red, blue, green-yellow, yellow, and yellow-red all shifted the positions of their modal values. Blue-red moved toward the shorter wavelengths and all others moved toward longer wavelengths.

Changes in exposure duration produced regular changes in color-name functions. The first increase in duration increased the height, decreased the range, and generally smoothed out most functions. The modal values of yellow and its compounds were shifted toward red, but only at the lowest surround-luminance level. A second increase in duration was required to produce this shift at the two higher surround luminances. In addition, the consequent increase in the height rather than the breadth of the category.

These data do not present any clear pattern of a Bezold-Brucke shift with changed surround luminances or exposure durations. Although further work should be done, a tentative conclusion would seem to be that shifts in the color appearance of stimuli characteristic of Bezold-Brucke do not occur simply as a function of changing stimulus brightness.

The presence of tritanopia at reduced brightness or briefer exposures was not detected. This would have been demonstrated by a reduction in the blue response under these conditions. Only the blue-red responses seemed to have been reduced, and these only at the briefest exposures. Changed surround luminance appeared to have no influence on the short wavelength portion of the spectrum. This study does not, in the writers' opinion, rule out Kaiser's notion that when retinal illuminance $X$ time equals a constant, Os use a constant color name (Kaiser, 1968). Our study varied only the surround and not the stimulus luminance and was, therefore, not directed to studying this proposed reciprocity.

The failure to find evidence for a shift characteristic of Bezold-Brucke does not mean that regular patterns of color shifts were not found at all. Some systematic changes in color appearances did result when sirround luminance interacted with stimulus duration, but changes in surround luminance alone did not have any substantial influence on color position. Varying the exposure duration, however, did influence spectral location of hues. These changes occurred primarily at values longer than $500 \mathrm{~nm}$. Every color-response category shifted to longer wavelengths at longer stimulus exposures. The shift below yellow could have been accounted for by some temporal analog to a Bezold-Brucke effect, but yellow, yellow-red, and red also shifted regularly to longer wavelengths at longer exposures. This may represent a color shift totally separate from the one induced by luminance changes, and warrants further attention.

\section{REFERENCES}

BEARE, A. C. Color-name as a function of wave-length. American Journal of Psychology, 1963, 76, 248-256.

Bouman, M. A., \& WalraVen, P. L. Some colour naming experiments for red and green monochromatic lights. Journal of the Optical Society of America, 1959, 49, 1022.

BOYNTON, R. M., SCHAFER, W., \& NEUN, M. E. Hue-wavelength relation incasured by color-naming method for three retinal locations. Science, 1964, 145, 666-668.

KAISER, P. K. Color names of very small fields varying in duration and luminance. Journal of the Optical Society of America, 1968, 58, 849-852. 
LURIA, S. M. Color-name as a function of stimulus intensity and duration. American Journal of Psychology, 1967, 80, 14-27.

MARRIOTT, F. H. C. Color naming experiments and the two-quanta theory. Journal of the Optical Society of America, 1959, 49, 1022. WEITZMAN, D. O., \& KINNEY, J. A. S. Appearance of color for small, brief, spectral stimuli, in the central fovea. Journal of the Optical Society of America, 1967, 57, 665-670.

(Accepted for publication July 6, 1970.) 